are comorbidities of rheumatoid arthritis (RA) and are related to the inflammatory burden of the disease, as well as to certain clinical and genetic characteristics of the disease. There is controversy about the role of ACPA in the development of $\mathrm{AE}$ in these patients.

Objectives: To explore the relation between ACPA and RF titres and subclinical vascular damage in RA patients.

Methods: Descriptive cross-sectional study with analytical components. A total of 244 RA patients were recruited consecutively over a period of 18 months (2013-2014) in a rheumatology department of a tertiary hospital. Patients with high vascular risk (vascular ischemic events, renal failure and diabetes mellitus) were excluded. Demographics, clinical data (ACPA and RF titres, duration of disease, hypertension and dyslipidemia) and vascular damage (atheroma plaque, carotid intima thickness [IMTc] and pulse wave velocity [PWV]) were collected. The atheroma plaque and IMTc measurement was performed by ultrasonography of the carotid arterial tree using an Esaote ${ }^{\circledR}$ MyLab70XVG with a 7-12 MHz linear transducer and an automated program measuring intima-media thickness (IMT) through radiofrequency (Quality intima media Thickness in real time, QIMT). PWV was obtained by analysis of brachial pulse waves with an automated and validated system (Mobil O Graph ${ }^{\circledR}$ ). Statistical analysis was performed with the SPSS 17.0 program.

Results: We included 181 patients, $141(77.9 \%)$ women and $40(22.1 \%)$ men, with a mean age of 60.4 years (SD 13.2) and a mean duration of the disease of 13.1 years (SD 10.5); 107 patients $(59.1 \%)$ were smokers or ex-smokers, $56(30.9 \%)$ hypertensive and $77(42.5 \%)$ dyslipemic. 118 patients $(65.2 \%)$ had positive ACPA, with a mean value of 330 (SD 621.5), and 107 (59.1\%) had a positive value of RF with a mean of 114 (SD 164.5)

No association was found between the positivity of ACPA and RF and the presence of atheroma plaques or with the values of IMTc and/or PWV. In patients with positive ACPA, a positive correlation was observed between ACPA titers and PWV values $(p<0.05)$. In particular, ACPA titers over 1600 were the ones that discriminated the highest values of PWV in our population $(p<0.009)$. The ACPA and RF values, on the other hand, were not related to the presence of plaques or to IMTc.

Conclusions: No relation was found between the positivity of RF and ACPA titers and the presence of pathological carotid ultrasound in our RA patient population. However, higher arterial stiffness was observed in patients with high ACPA titers. Disclosure of Interest: None declared

DOI: 10.1136/annrheumdis-2017-eular.3245

\section{AB0345 PSYCHIATRIC DISORDERS RELATED TO HYPERLEPTINEMIA AND RHEUMATOID ARTHRITIS CLINICAL ACTIVITY MEASURED BY DAS28}

M. Vázquez-Del Mercado ${ }^{1,2}$, A.-M. Rodrígez-Ramírez ${ }^{2}$, E. Chavarria-Avila ${ }^{2}$, F. Sandoval-García ${ }^{2}$, B.-T. Martin-Marquez ${ }^{2}$, R.-E. Navarro-Hernandez ${ }^{2}$, F. Corona-Meraz ${ }^{2}$, E. Gomez-Bañuelos ${ }^{2} .{ }^{1}$ Servicio de Reumatología, División de Medicina Interna, Pnpc 004086, CONACYT, Hospital Civil Juan I. Menchaca;

${ }^{2}$ Instituto de Invesgitación en Reumatología y del Sistema Musculoesquelético, Universidad de Guadalajara, Guadalajara, Mexico

Background: Leptin is an adipose-derived hormone with a role in depression related to chronic stress, anxiety and pain disorders, disturbs with a high prevalence in RA patients. In previous studies, it has been demonstrated the role of leptin in the pathogenesis of RA, in particular, its association with anti-CCP. The purpose of this study was to evaluate the psychiatric disorders risk related to serum soluble leptin (sLep) levels with clinical activity in RA patients.

Objectives: To evaluate the association between psychiatric disorders and serum leptin (sLep) levels and RA disease activity

Methods: 76 outpatients diagnosed with RA (ACR1987/ ACR/ EULAR2010) were evaluated with clinical, laboratory and image assessment. Disease activity was measured using DAS28 CRP, the Mini International Neuropsychiatric Interview Plus (M. I. N. I. Plus) was used for the psychiatric evaluation. sLep levels were measured by ELISA method.

Results: sLep levels were significantly higher in RA patients M.I.N.I. plus (+) $(P<0.001)$. DAS28 CRP score was significantly higher in patients with a present psychiatric disorder $(P=0.006)$ with an odds ratio of $1.6([1.04-2.46] P=0.03)$ for present psychiatric comorbidity. After adjustment with age and years of RA diagnosis, the odds ratio increased to $1.72([1.08-2.73] P=0.02)$. Also, DAS28 CRP had a moderate correlation with the number of psychiatric diagnosis present, past and for lifetime ( $\mathrm{r}=0.485, P<0.001, \mathrm{IC} 95 \%$ [0.99-0.636]).

Conclusions: sLep levels and DAS28-CRP were associated with symptoms of depression, such as anxiety and pain disorder in RA patients.

Disclosure of Interest: None declared

DOI: 10.1136/annrheumdis-2017-eular.6808

\section{AB0346 MOOD DISORDERS (ANXIETY AND DEPRESSION) IN RHEUMATOID ARTHRITIS}

M. Brahem ${ }^{1}$, M. Maraoui ${ }^{1}$, H. Hachfi ${ }^{1}$, S. Ben Hammouda ${ }^{1}$, I. Haddada ${ }^{1}$ M. Jguirim ${ }^{2}$, M. Younes ${ }^{1}$. ${ }^{1}$ Rheumatology, Taher Sfar Hospital Mahdia, mahdia; ${ }^{2}$ Rheumatology, Fattouma Bourguiba Hospital Monastir, Monastir, Tunisia

Background: In addition to recurrent pain, fatigue, and increased rates of physical disability, individuals with rheumatoid arthritis (RA) have an increased prevalence of some mental health disorders, particularly those involving affective or mood disturbances.Many researchers have shown that mood disturbance and disability may serve as important pathways through which disease burden contributes to poor health functioning in RA.

Objectives: Our aim is to estimate the mood disorders (anxiety and depression) in patients with RA and to evaluate the associated factors.

Methods: This is a cross-sectional and descriptive study during a period of the year 2016, including 37 patients followed in the department of Rheumatology in Mahdia Tunisia. All patients were diagnosed with RA based in ACR 1987/EULAR2010.We evaluated for each patient the parameters of activity of the disease, the quality of life by the HAQ questionnaire and the mood disorders using the Hospital Anxiety and Depression Scale (HAD).

Results: The age of the RA patients (32 females/5 males) ranged from 21 to 76 years. The mean age was $53.1 \pm 12$ years. The mean duration of the disease was $11 \pm 10$ years [1-34]. The mean number of tender joints was $13.2 \pm 9.6$ and swollen joint was $5.9 \pm 7$. The mean DAS28 was $5.5 \pm 1.5[2.9-8.2]$ and HAQ was $1.6 \pm 0.9$ [0-2.8]. 51.3\% of patients had specific joint deformations, $83.8 \%$ had radiologic involvement and $29.7 \%$ had osteoporosis.

The biologic analysis showed that the mean ESR was $45 \pm 27.1$ and the CRP was $13.7 \pm 25.3$. Rheumatoid factors were positive in $37.8 \%$ of cases, the ACPA were positive in $32.4 \%$ of cases. $81.1 \%$ of RA patients were treated by methotrexate and $13.5 \%$ were treated by biologic treatments.

The average score of depression was $9 \pm 2.6$ [1-14]. Basing on this score, 12 patients $(32.4 \%$ of cases) didn't have depression (score $<8), 14$ patients $(37.8 \%$ of cases) suffered probably from depression (score between 8 and 10) and 11 patients $(29.7 \%)$ had a certain depression (score $\geq 10$ ). Our study didn't found any correlation between the score of depression and the age, the score and clinical parameters of RA (duration of disease, DAS28, number of tender and swollen joints and deformations), the score and biologic parameters (ESR, CRP, Rheumatoid factor, ACPA).

The average score of anxiety was $9.2 \pm 4.2[0-20] .12$ patients (32.4\%) didn't have signs of anxiety (score $<8), 12$ patients $(32.4 \%$ ) had probably an anxiety (score between 8 and 10 ) and 13 patients (35.2\%) presented a certain anxiety $($ score $>10)$. We found a significant correlation between the score of anxiety and the number of tender joints, the anxiety score and the HAQ (Health Assessment Quality).

Conclusions: Our study showed that the majority of our RA patients suffered from mood disorders; $67.5 \%$ had signs of depression and $65.6 \%$ had signs of anxiety.So, it's important to evaluate the mood disturbances in RA patients to ameliorate their quality of life.

References:

[1] John A. Sturgeon et al, Affective disturbance in rheumatoid arthritis: psychological and disease-related pathways; reviews Rheumatology 2016.

[2] SARAH R. ORMSETH et al, Multidimensional Model of Disability and Role Functioning in Rheumatoid Arthritis; Arthritis Care \& Research, Vol. 67, No. 12, December 2015, pp 1686-1692.

Disclosure of Interest: None declared

DOI: 10.1136/annrheumdis-2017-eular.5279

\section{AB0347 EVALUATION OF THE IMPACT OF RHEUMATOID ARTHRITIS ON SEXUAL FUNCTION}

M. Brahem ${ }^{1}$, H. Hachfi ${ }^{1}$, S. Ben Hammouda ${ }^{1}$, I. Haddada ${ }^{1}$, M. Maraoui ${ }^{1}$,

M. Jguirim ${ }^{2}$, M. Younes ${ }^{1} .{ }^{1}$ Rheumatology, Taher Sfar Hospital Mahdia, mahdia; ${ }^{2}$ Rheumatology, Fattouma Bourguiba Hospital Monastir, Monastir, Tunisia

Background: Rheumatoid arthritis (RA) may affect all aspects of life including sexual functioning. The percentage of arthritic patients who experience sexual problems ranged in various studies from $31 \%$ to $76 \%$. The reasons for disturbing sexual functioning are multi-factorial and comprise disease-related factors as well as therapy.It can occur before, during and after sexual activities, and can affect sexual health in different perspectives.

Objectives: The aim of our study is to evaluate the impact of the RA in sexual function and its associated factors.

Methods: This is a cross-sectional and descriptive study during a period of the year 2016, including 37 patients followed in the department of Rheumatology in Mahdia Tunisia. All patients were diagnosed with RA based in ACR 1987/EULAR 2010. We evaluated for each patient the sexual quotient (QS). It is a validated scale which estimates the degree of satisfaction of the sex life.

Results: The age of the RA patients (32 females/5 males) ranged from 21 to 76 years. The mean age was $53.1 \pm 12$ years. The mean duration of the disease was $11 \pm 10$ years [1-34]. The mean number of tender joints was $13.2 \pm 9.6$ and swollen joint was $5.9 \pm 7$. The mean DAS28 was $5.5 \pm 1.5$ [2.9-8.2] and HAQ was $1.6 \pm 0.9$ [0-2.8]. 51.3\% of patients had specific joint deformations, $83.8 \%$ had radiologic involvement and $29.7 \%$ had osteoporosis.

The biologic analysis showed that the mean ESR was $45 \pm 27.1$ and the CRP was $13.7 \pm 25.3$. Rheumatoid factors were positive in $37.8 \%$ of cases, the ACPA were positive in $32.4 \%$ of cases. $81.1 \%$ of RA patients were treated by methotrexate and $13.5 \%$ were treated by biologic treatments.

The mean sexual quotient was $50.25 \pm 20.8$ [6-87.5]; only $8.1 \%$ of patients had a great sexual life (QS between 80 and 100), 16.2\% expressed some sexual satisfaction (QS between 60 and 80 ), 21.6\% had a mild sexual life (QS between 
40 and 60), 18.9\% presented a disappointing sexual life (QS between 20 and 40) and $35.4 \%$ didn't have any partner.

Our study showed a significant correlation between the duration of the disease and the alteration of the sexual quotient, but no correlation with the clinical and biological parameters of RA.

Conclusions: We conclude that only $24.3 \%$ of our RA patients had satisfaction in their sexual life. This alteration of the sexual quotient was found to be correlated with the duration of the disease.

This fact may be explicated by the disability, fatigue and pain caused by this chronic inflammatory disease. This relation should be confirmed by future studies. References:

[1] Antonio G Tristano, Impact of rheumatoid arthritis on sexual function, World J Orthop 2014 April 18; 5(2): 107-111.

[2] Abdo J. Sex Med. 2007;4:382-389.

[3] Kraaimaat FW, et al, Intrusiveness of rheumatoid arthritis on sexuality in male and female patients living with a spouse. Arthritis Care Res 1996; 9:120-125. Disclosure of Interest: None declared

DOI: 10.1136/annrheumdis-2017-eular.5298

\section{AB0348 SLEEP DISORDERS IN PATIENTS WITH RHEUMATOID ARTHRITIS}

M. Brahem ${ }^{1}$, S. Ben Hammouda ${ }^{1}$, H. Hachfi ${ }^{1}$, M. Maraoui ${ }^{1}$, I. Haddada ${ }^{1}$

M. Jguirim ${ }^{2}$, M. Younes ${ }^{1} .{ }^{1}$ Rheumatology, Taher Sfar Hospital Mahdia, mahdia;

${ }^{2}$ Rheumatology, Fattouma Bourguiba Hospital, Monastir, Tunisia

Background: Rheumatoid arthritis (RA)as one of the most common autoimmune diseases is known to be one of the leading causes of disability.Sleep disorders have direct influence on patient's life. But the exact nature of relationship between sleep disorders and Rheumatoid arthritis is not completely understood.

Objectives: The aim of our study is to evaluate the impact of RA in sleep quality and to establish associated factors.

Methods: This is a cross-sectional and descriptive study during a period of the year 2016, including 37 patients followed in the department of Rheumatology in Mahdia Tunisia. All patients were diagnosed with RA based on ACR 1987/EULAR2010. We evaluated for each patient the parameters of activity of the disease, the quality of life by the HAQ questionnaire and the quality of sleep using two scales: Epworth (ESS) and Pittsburg scale (PSQI) which is composed from 7 components rated each one from 0 to 3 .

Results: The age of the RA patients (32 females/5 males) ranged from 21 to 76 years. The mean age was $53.1 \pm 12$ years. The mean duration of the disease was $11 \pm 10$ years [1-34]. The mean number of tender joints was $13.2 \pm 9.6$ and swollen joint was $5.9 \pm 7$. The mean DAS28 was $5.5 \pm 1.5[2.9-8.2]$ and HAQ was $1.6 \pm 0.9$ [0-2.8]. $51.3 \%$ of patients had specific joint deformations, $83.8 \%$ had radiologic involvement and $29.7 \%$ had osteoporosis.

The biologic analysis showed that the mean ESR was $45 \pm 27.1$ and the CRP was $13.7 \pm 25.3$. Rheumatoid factors were positive in $37.8 \%$ of cases, the ACPA were positive in $32.4 \%$ of cases. $81.1 \%$ of RA patients were treated by methotrexate and $13.5 \%$ were treated by biologic treatments.

The mean Epworth score was $9 \pm 5.7(0-23) .56 .8 \%$ of patients had no sleep debt, $32.4 \%$ had a sleep deficit and only $10.8 \%$ had signs of somnolence. Our study confirmed a significant correlation between the Epwoeth score and the number of tender joints, the ESR, the Health assessment quality (HAQ) score.

Regarding the overall score of Pitsburg, the average was $8.4 \pm 4.1$ (1-16). The average of the "subjective sleep quality" was 1.35 , "latency to sleep" was 1.81 , "sleep duration" was 1.24, "habitual sleep efficiency" was 1.08, "sleep disorders" was 1.62, "the use of a sleep medicine" was 0.27 and finally the average of the 7th component about "poor form during the day" was 1.11 out of 3 . So the latency to sleep and sleep disorders were the most affected components. We had a significant correlation between PSQI and the number of swollen joints, the $\mathrm{HAQ}$ score. The value of the ACPA was found to be associated with high score of PSQI.

Conclusions: Our study showed that the sleep disruption wasn't rare in patients with RA. This can be related to the disability and pain caused by this disease. Further studies with large sample size, as well as more careful tools of sleep disorders, would help to generalize results and suggestions. By providing adequate health care, and recognition of the patients' pain conditions we would ameliorate sleep quality and increase the QOL of RA patients.

References:

[1] Majid Purabdollah, et al, Relationship between Sleep Disorders, Pain and Quality of Life in Patients with Rheumatoid Arthritis; Journal of Caring Sciences, 2015, 4(3), 233-241.

[2] Seda PEHLIVAN et al, Sleep quality and factors affecting sleep in elderly patients with rheumatoid arthritis in Turkey; Turk J Med Sci (2016) 46: 1114-1121.

Disclosure of Interest: None declared

DOI: 10.1136/annrheumdis-2017-eular.5251

\section{AB0349 THE SERUM LEVEL OF IRISIN IS DECREASED IN THE PATIENTS WITH RHEUMATOID ARTHRITIS}

A.T. Kalkan ${ }^{1}$, M. Ozmen ${ }^{2}$, B. Birlik ${ }^{3}$, D. Kozaci ${ }^{4}$, I. Turkmen ${ }^{5}$, D. Solmaz ${ }^{2}$, O. Gercik ${ }^{2}$, S. Gucenmez ${ }^{2}$, S. Akar ${ }^{2} .{ }^{1}$ Department of Internal Medicine; ${ }^{2}$ Department of Internal Medicine, Division of Rheumatology; ${ }^{3}$ Department of Radyology, Izmir Katip Celebi University, School of Medicine; ${ }^{4}$ Department of Medical Biochemistry; ${ }^{5}$ Department of Musculoskeletal System and

Regenerative Medicine, Ankara Yıldırım Beyazıt University, School of Medicine, Izmir, Turkey

Background: Adipomyokines are proteins that are synthesized by and secreted from both skeletal muscle and adipose tissue, and show their effects through autocrine, paracrine or endocrine pathways (1). Irisin, a novel adipomyokine, is secreted in association with exercise from the skeletal muscle, and from the white fat tissue to help the brown fat tissue gain the energy expenditure phenotype (2). There is evidence that the irisin is associated with metabolic syndrome (MetS) and cardiovascular risk $(3,4)$.

Objectives: Rheumatoid arthritis (RA) is associated with an increased risk of cardiovascular disease (CVD) and MetS compared with the general population $(5,6)$. The aims of this study were to assess the serum level of irisin, and the possible relationships of irisin with disease activity in patients with RA.

Methods: Eighty four consecutive RA patients fulfilling the 2010 ACR/EULAR RA Classification Criteria were included in the study. Fifty age- and sexmatched healthy volunteers were enrolled as the control group. Disease duration, medications, history of traditional risk factors of CVD and demographic data of patients were noted. Body Mass Index (BMI) was calculated as "weight $(\mathrm{kg}) /$ height $(\mathrm{m})^{2}$ ". HbA1c, lipid profile, insulin were measured. Insulin resistance was assessed with the Homeostasis Model Assessment (HOMA) Index. RA disease activity was assessed by disease activity score based on evaluation of 28 joints (DAS28). Serum irisin level was assessed by ELISA. Measurement of carotid intima media thickness by carotid doppler ultrasonography was performed by a radiologist for cardiovascular risk assessment.

Results: There was no significant difference between the groups in terms of BMI $(p=0,20), \operatorname{HbA1c}(p=0,15)$, lipid profiles $(p<0.05)$, insulin resistance $(p=0.72)$ and carotid intima-media thickness $(p=0.216)$.

Serum irisin levels were found to be significantly lower in RA patients $(20,65$ (minimum:16,94- maximum:99,35) ng/mL) than healthy controls $(37,56(18,37-$ $84,70) \mathrm{ng} / \mathrm{mL})(\mathrm{p}<0.001)$. There was no relationship between RA disease activity and irisin levels.

Conclusions: This study showed that irisin was significantly lower than controls. Irisin may be responsible for increased cardiovascular risk in RA patients. But before a definite judgment; prospective studies with a larger sample size assessing the exercise behaviour of patients and the presence of CVD are necessary.

\section{References:}

[1] Raschke S, Eckel J. Adipo-myokines: two sides of the same coin - mediators of inflammation and mediators of exercise. Mediators Inflamm. 2013;320724:116.

[2] Roca-Rivada A, et al. FNDC5/irisin is not only a myokine but also an adipokine. PLoS One. 2013; 8(4):e60563.

[3] Yan B, et al. Association of serum irisin with metabolic syndrome in obese Chinese adults. PLoS One. 2014 Apr 7;9(4):e94235.

[4] Deng W. Association of Serum Irisin Concentrations with Presence and Severity of Coronary Artery Disease. Med Sci Monit. 2016 Nov 5;22:41934197.

[5] Wolfe F,et al. Increase in cardiovascular and cerebrovascular disease prevalence in rheumatoid arthritis. J Rheumatol 2003;30: 36-40.

[6] Ozmen M, et al. Prevalence of the metabolic syndrome in rheumatoid arthritis. Eur J Rheum 2014; 1: 1-4.

Disclosure of Interest: None declared

DOI: 10.1136/annrheumdis-2017-eular.2040

\section{AB0350 FUNCTIONAL DISABILITY MEASURED BY HEALTH ASSESSMENT QUESTIONNAIRE (HAQ) CORRELATES WITH DISEASE ACTIVITY IN ELDERLY RHEUMATOID ARTHRITIS PATIENTS}

N. Alpay-Kanıtez ${ }^{1}$, Ö. Pehlivan ${ }^{2}$, A. Omma ${ }^{3}$, S. Can-Sandıkçı ${ }^{3}$ S. Yılmaz-Öner ${ }^{1}$, C. Bes ${ }^{1}$, S. Çelik ${ }^{1}$. ${ }^{1}$ Bakırköy Dr. Sadi Konuk' Research and Training Hospital; ${ }^{2}$ Umraniye Research and Training Hospital, Istanbil; ${ }^{3}$ Ankara Numune Research and Training Hospital, Ankara, Turkey

Background: Rheumatoid arthritis (RA)'s prevalence increases with age and the recognition of functional disability related to RA could be challenging in elderly patients (1).

Objectives: In this study, we aimed to look at the correlation between disease activity and the functional disability by using HAQ score in elderly RA patients.

Methods: Elderly RA patients, $\geq 65$ years old at their routine visits were included to the study. The composite "disease activity score" in 28 joints (DAS-28) and "Physician Global Assessment" (PhGA) were used to determine disease activity. Health assessment questionnaire (HAQ) scores were calculated to describe the functional disability and compared across the disease activity groups according to DAS-28.

Results: Two hundred and fifty eight RA patients with the mean age of $71 \pm 5$ 